\section{ASPECTOS PSICOLÓGICOS ESPECÍFICOS DEL CÁNCER EN LAS MUJERES}

\author{
María Die Trill \\ Hospital Universitario Gregorio Marañón \\ mdietrill@gmail.com
}

Cómo citar este artículo/Citation: Die Trill, M. (2015). "Aspectos psicológicos específicos del cáncer en las mujeres". Arbor, 191 (773): a240. doi: http://dx.doi.org/10.3989/ arbor.2015.773n3010

Recibido: 9 diciembre 2014. Aceptado: 24 abril 2015.

RESUMEN: Las diferencias en los aspectos psicosociales de diferentes tipos de cáncer en hombres y mujeres están basadas fundamentalmente en variables de género. Sin embargo, la forma concreta en la que influye el género en la adaptación al cáncer no está del todo definida, y desde luego, muy poco estudiada. Las diferencias de género no solo son evidentes en el impacto físico del cáncer sino también en la calidad de vida, en las diferencias psicosociales, en los estilos de afrontamiento y en la adaptación de las parejas de los pacientes al proceso de enfermedad. Es necesario identificar con más detalle las necesidades psicosociales específicas de la mujer con el fin de poder desarrollar programas concretos que tengan en cuenta las diferencias de género en el afrontamiento de la enfermedad oncológica, en el proceso de toma de decisiones médicas, y en las variables necesarias para la optimización de su calidad de vida.

PALABRAS CLAVE: afrontamiento; imagen corporal; maternidad; sexualidad.

\section{SPECIFIC PSYCHOLOGICAL ASPECTS OF CANCER IN WOMEN}

Copyright: (c) 2015 CSIC. Este es un artículo de acceso abierto distribuido bajo los términos de la licencia Creative Commons Attribution-Non Commercial (by-nc) Spain 3.0.

ABSTRACT: The differences in the psychosocial aspects of different types of cancer in men and women are mainly based on gender variables. However, the specific way in which gender influences adaptation to cancer is not completely defined and it has also been very poorly studied. Gender differences are not only evident in the physical impact of cancer, but also in quality of life, in the psychosocial differences, in the confronting styles and in the adaptation of the patient's partners to the process of the disease. It is necessary to identify women's specific psychosocial needs in more detail in order to be able to develop specific programs which keep in mind the gender differences in the way oncologic disease is confronted, as well as in the process of making clinical decisions and in the variables required to optimise their quality of life.

KEYWORDS: body image; confronting; maternity; sexuality. 


\section{INTRODUCCIÓN}

Ser varón o hembra es una variable humana básica fundamental que afecta a la salud y a la enfermedad a lo largo del ciclo vital (Wizemann y Pardue, 2001). Los hombres y las mujeres no solo difieren en cuanto a sus cuerpos y sus órganos reproductores, sino también en la forma en la que piensan, sienten y se comportan. A las diferencias físicas nos referimos como sexo, y están influenciadas por los genes y la biología. A las diferencias psicológicas nos referimos como género, y en él juegan un rol significativo los factores psicosociales, ambientales y culturales. Los investigadores están intentando desengranar las complejas interacciones entre el sexo y el género, no estando aún del todo claros los roles que juegan la naturaleza y la crianza sobre ellas (Kiss y Meryn, 2001).

En el caso del cáncer es evidente que algunos tipos de cáncer son exclusivos del varón (por ejemplo, el cáncer de próstata) o de la mujer (por ejemplo, el cáncer de ovario). En otros tipos de cáncer sin embargo, la predominancia que ha sido específica de un género, actualmente está sufriendo cambios significativos (por ejemplo, el cáncer de pulmón). Las diferencias en los aspectos psicosociales de diferentes tipos de cáncer en hombres y mujeres están basadas fundamentalmente en variables de género. Sin embargo, la forma concreta en la que influye el género en la adaptación al cáncer no está del todo definida (Kiss y Meryn, 2001), y desde luego, muy poco estudiada. Las diferencias de género no solo son evidentes en el impacto físico del cáncer sino también en la calidad de vida, en las diferencias psicosociales, en los estilos de afrontamiento y en la adaptación de las parejas de los pacientes al proceso de enfermedad.

En este artículo se describirá el impacto psicosocial que tiene el cáncer sobre diferentes áreas de funcionamiento de la mujer, incluyendo los estilos de afrontamiento y el uso del apoyo social que hacen las mujeres con cáncer, la imagen corporal y la femineidad, la sexualidad y sus alteraciones a consecuencia de la enfermedad y sus tratamientos, cuestiones relacionadas con la maternidad e infertilidad, las relaciones materno-filiales y el impacto del cáncer materno sobre los hijos, y aspectos específicos del riesgo genético sobre la mujer con cáncer.

\section{ESTILOS DE AFRONTAMIENTO, APOYO SOCIAL Y CÁNCER EN LA MUJER}

Aunque cada día estas diferencias se difuminan más, la literatura aún contempla la idea de que mien- tras las mujeres suelen ser mejores comunicadoras, los varones, por regla general, tienden a compartimentalizar. En momentos difíciles, las mujeres necesitan hablar sobre las dificultades por las que están pasando, mientras que los varones parecen preferir no hacerlo. Se ha sugerido que con el fin de comprender la vida de las mujeres, uno debe de entender los tipos de estrés que sufren y las maneras que tienen de afrontarlo y que son específicas a su proceso de socialización (Tom, 1993). Estos conceptos estereotipados pueden aplicarse al proceso oncológico. Así, se ha comprobado que durante momentos de angustia, las mujeres con cáncer de mama necesitan hablar sobre su experiencia de la enfermedad con otras personas y compartir sus emociones al respecto, en oposición a varones con cáncer de próstata, que prefieren no hacerlo (Volkers, 1999).

Aunque existen pocos estudios específicos al cáncer, algunas investigaciones reportan que con frecuencia, las mujeres se enfrentan al cáncer de manera más adaptativa que los varones, tanto si son pacientes como si son cuidadoras. Esto quizás pueda atribuirse, entre otras cosas, a que las mujeres cuentan con mayores sistemas de apoyo social que los hombres (Volkers, 1999). Redes sociales mayores han sido asociadas a la presencia de menos síntomas depresivos y a una menor alteración del estado afectivo en pacientes con cáncer (Grassi et al., 1997), y una percepción de mayor apoyo social eficaz se ha asociado a depresión menos severa y a mejor calidad de vida en esta población (Lee, 1997). En un estudio realizado por Hann y sus colegas (Hann et al., 2002), se observó que una mayor red de apoyo social estaba asociada a depresión menos severa en mujeres con cáncer, pero no en varones.

El hecho de que las mujeres necesiten comunicarse por regla general, más que los varones se refleja igualmente en la mayor participación de estas en grupos de apoyo para pacientes o supervivientes de cáncer. Klemm estudió tres diferentes tipos de grupos de apoyo vía internet (Klemm, 2012; Klemm et al., 2003): un grupo con varones con cáncer de próstata en el que participaron 117 pacientes; un grupo con 126 mujeres con cáncer de mama, y un tercer grupo mixto, formado por 92 hombres y mujeres con cualquier tipo de cáncer. En el grupo mixto, tanto los hombres como las mujeres tendían a ofrecer y solicitar información médica, pero cuando se les separó en el grupo, se encontraron diferencias en el comportamiento entre ambos grupos de género. En el grupo de mujeres, la mayor parte de los comentarios giraban en torno a 
los sentimientos que estaban experimentando con la enfermedad. Los varones en cambio, o bien preguntaban cuestiones concretas sobre el cáncer o la quimioterapia, o bien ofrecían información sobre estos dos aspectos de la enfermedad. Las mujeres tendían más a animarse mutuamente y a compartir experiencias personales con el cáncer, como también lo demuestra la experiencia clínica.

Se ha estudiado cómo se enfrentan las mujeres al diagnóstico oncológico y si su comportamiento en este momento del proceso de enfermedad difiere del de los varones. Sloan observó que mujeres con cáncer de mama fueron más asertivas durante la confirmación del diagnóstico oncológico comparadas con otros grupos de pacientes (Sloan, 1992), siendo los hombres con cáncer de próstata los menos asertivos. Una posible explicación que el investigador aporta es que las mujeres están acostumbradas a hablar con los profesionales de la salud sobre cuestiones relacionadas con el sistema reproductor desde que son muy jóvenes $y$, por tanto, pueden sentirse más cómodas hablando sobre la salud en general, que los varones.

\section{IMAGEN CORPORAL Y FEMINEIDAD}

Teorías sobre la evolución y socioculturales demuestran que el género es un factor determinante del grado de satisfacción que tiene una persona con su cuerpo (Algars et al., 2009). La investigación apoya la creencia de que las mujeres difieren de los hombres en su perspectiva sobre la imagen corporal (Li y Rew, 2010). A pesar de que tanto hombres como mujeres se preocupan por esta, se ha sugerido que se deben tener en cuenta las diferencias de género cuando se estudie este concepto de imagen corporal (Woertman y van den Brink, 2012).

En el caso de mujeres recibiendo tratamiento oncológico, fuentes importantes de identidad femenina (la melena, las mamas, etc) pueden verse alteradas o extirpadas, lo cual puede producir un elevado distress y una sensación significativa de insatisfacción con su propio cuerpo (Ashing-Giwa et al., 2004). Igualmente, se ha observado que mujeres que se someten a resecciones abdominoperineales reportan peor imagen corporal que los varones, y menor actividad sexual que estos después de la cirugía (Tekkis et al., 2009). Una imagen corporal más negativa en mujeres que en varones con cáncer se han visto documentada en otros estudios (Krok, Baker y McMillan, 2013), con casi el $90 \%$ de mujeres reportando preocupaciones significativas con su peso (Cash, Melnyk y Hrabosky, 2004). Además, el distress generado por la toxicidad terapéutica asociada a la imagen corporal (por ejemplo, alopecia) es con frecuencia valorado por las mujeres como más intenso que el asociado a otras secuelas del tratamiento por ejemplo, náuseas, astenia, etc. (Liu, 2010).

El impacto emocional que produce la extirpación de órganos internos asociados a la femineidad o a aspectos importantes de la condición femenina, como la maternidad, puede ser igualmente significativo sobre la mujer. Tal es el caso de aquéllas en las que haya que practicar una histerectomía, ooforectomía o cualquier otra cirugía que afecte a su capacidad reproductora y/o a su función sexual, haciéndolas sentir con frecuencia, "menos mujer" y "menos madre", a pesar de que la alteración física es interna y no visible.

La alteración en la imagen corporal y en el concepto de femineidad que producen el cáncer y sus tratamientos dependerá en gran parte, del significado que la mujer atribuya al órgano/s afectado/s. Si una mujer basa su autoestima fundamentalmente en su aspecto físico, incluso una pequeña cicatriz quirúrgica puede producir un nivel elevado de angustia. Para una cuya femineidad esté estrecha y casi exclusivamente vinculada a las mamas, cuando se tenga que someter a una mastectomía sufrirá enormemente. Sin embargo una mujer cuya autoestima esté basada no solo en su aspecto físico sino en otras cualidades (por ejemplo en su capacidad de amar, de mantener relaciones, de crear, etc), no sufrirá con la misma intensidad cuando experimente alteraciones físicas como consecuencia del cáncer.

Es también importante averiguar el significado que tienen los órganos femeninos para la mujer para entender el proceso de decisiones médicas en el que se involucra. Así, mientras que para una mujer con un tumor mamario puede ser muy importante preservar su mama y someterse a un tratamiento quirúrgico conservador, para otra con la misma condición, puede ser más importante someterse a una mastectomía por considerar que este procedimiento quirúrgico le ayudará a sentirse "más limpia" del cáncer. Mientras que para una paciente perder el pelo con la quimioterapia puede ser fuente de enorme angustia y evitación de contactos sociales, para otro, la alopecia es "algo secundario" porque "lo que realmente me interesa es salvar mi vida, y el pelo, ya me crecerá".

Entre las reacciones psicológicas más frecuentes en mujeres con cáncer que sufren alteraciones en su imagen corporal se pueden mencionar un gran temor al rechazo por personas del entorno, lo cual conduce a 
las pacientes en ocasiones a la evitación de relaciones sociales y sexuales, produciéndose en ocasiones un aislamiento y soledad que conllevan dificultades para la reintegración social después del cáncer. Las alteraciones en la imagen corporal pueden causar ansiedad interpersonal y distanciamiento de los otros. Pensamientos del tipo: "Si yo no me gusto, no me acepto, no podré gustar a los demás" son por desgracia, frecuentes, especialmente en una sociedad en la que el aspecto físico está sobrevalorado. Alteraciones emocionales frecuentes asociadas a los cambios en la imagen corporal incluyen ansiedad, angustia, depresión, tristeza, impotencia y rabia (Die Trill, 2004).

\section{SEXUALIDAD}

El cáncer o sus tratamientos pueden afectar la sexualidad de la mujer que lo padece a través de cuatro vías principales:

1. Produciendo problemas físicos que impiden dar y recibir placer sexual;

2. Produciendo alteraciones en la imagen corporal, ya descritas anteriormente;

3. Generando sentimientos tales como el miedo, la angustia, la tristeza, etc., que pueden interferir con una respuesta sexual adecuada, y/o

4. Produciendo modificaciones en los roles y relaciones que mantiene la paciente.

La relación entre estas cuatro áreas es de tal importancia que si se altera una, pueden verse afectadas las demás. En el caso del cáncer de mama, la pérdida de una mama o de una parte de ella puede dañar significativamente el sentido de atractivo que siente una mujer. Las mamas y los pezones son una fuente de placer para la mujer y perderlos puede resultar una experiencia traumática para algunas. Algunas mujeres mastectomizadas sufren dolores de espalda tras la cirugía y han de utilizar almohadas para acomodarse durante la relación sexual. $\mathrm{Ni}$ el cáncer de mama per se, ni su tratamiento con cirugía o radioterapia deben producir alteraciones en el deseo sexual ni reducir su capacidad para lubricar, tener sensaciones genitales placenteras o alcanzar un orgasmo. Son más bien factores psicológicos y el significado que se le atribuye a la mama (femineidad, capacidad reproductora, maternidad, atractivo, etc.), junto a algunos efectos secundarios de la quimioterapia y hormonoterapia (cuando se tengan que administrar), los que pueden alterar la respuesta sexual. Las cirugías reconstructoras suelen aliviar los efectos psicológicos del tratamiento quirúrgico en las mujeres que se someten a ellas.
En los casos de cáncer de cérvix, generalmente las alteraciones sexuales que pueden surgir se deben con mayor frecuencia a los efectos de la radioterapia que a los de la cirugía. Esto es debido a la fibrosis, estenosis vaginal, y reducción en la lubricación que produce esta modalidad terapéutica. Las alteraciones sexuales en estos casos se pueden tratar en gran parte a través de programas de educación sexual, apoyo psicológico, uso de lubricantes, y cambios de postura durante la relación sexual, entre otras cosas. Igual que en el caso del cáncer de mama y otros tumores propios de la mujer, el sentido de la femineidad, maternidad, capacidad reproductora, etc. de la enferma pueden verse alteradas, repercutiendo en sus relaciones sexuales (Amsterdam y Krychman, 2006).

Un porcentaje elevado de mujeres con cáncer de ovario y endometrio reportan dificultades sexuales y ausencia de relaciones sexuales. El deseo sexual puede desaparecer en estas mujeres, aunque es recuperable (Carmack Taylor et al., 2004). Aunque el cáncer de vulva es poco frecuente y aparece a edades avanzadas, frecuentemente genera sentimientos de depresión y aislamiento. Aún así y a pesar de la agresión sufrida, la mujer afectada puede experimentar deseo sexual y debe encontrar maneras alternativas de poder satisfacerlo.

En general, los avances de las técnicas quirúrgicas han ido produciendo una menor morbilidad sexual en las mujeres con cáncer. La histerectomía, la mastectomía, la resección abdominoperineal, la cistectomía radical, la vulvectomía y la resección pélvica total constituyen los tratamientos quirúrgicos que producen alteraciones sexuales con mayor frecuencia. En muchos casos, efectos secundarios de la quimioterapia tales como las náuseas y los vómitos, la mucositis, las alteraciones perceptivas del gusto y del tacto, la diarrea y el estreñimiento, los cambios en la imagen corporal (p.e., cambios en la textura de la piel, alopecia, cambios en el peso, caída del vello púbico, etc.) generan un importante sentimiento de asexualidad. A este desagradable sentimiento se añade, en el caso de la mujer con cáncer, sequedad vaginal, dispareunia (dolor durante el coito), mayor dificultad para alcanzar un orgasmo y posibles síntomas menopáusicos. Además, la privación de estrógenos puede producir atrofia vaginal, estrechez de los tejidos vulvares y vaginales, infecciones urinarias más frecuentes y labilidad afectiva, cansancio e irritabilidad.

Estas secuelas tienen difícil tratamiento en ocasiones, especialmente cuando la administración de estrógenos está contraindicada por el tipo de tumor 
diagnosticado. El cansancio, las náuseas y vómitos, la diarrea y la irritación de la piel irradiada secundarios a la administración de radioterapia afectan adversamente al funcionamiento sexual de la mujer que recibe este tratamiento, que puede provocar disfunciones sexuales prolongadas, exámenes vaginales dolorosos, dispaurenia e infertilidad. Los cambios producidos por la radioterapia en la función sexual suelen ser lentos y pueden mejorar con el paso del tiempo (Diaz Morfa, 2008).

Las alteraciones de la imagen corporal secundarias a la enfermedad pueden también afectar significativamente a la función sexual, como se ha descrito previamente. El dolor puede producir una disminución del deseo sexual, tanto si es dolor experimentado en los genitales durante el coito como si es dolor en un órgano no sexual del cuerpo. Asimismo, el dolor puede distraer de la sensación placentera durante el contacto sexual. Tal es el caso de mujeres que sufren linfedema con las consecuentes molestias en el brazo tras haber sufrido una mastectomía.

El papel que juegan la cercanía afectiva y la intimidad en la adaptación sexual está siendo cada vez más reconocido en Oncología. Existe evidencia de que para las mujeres, la satisfacción sexual está más fuertemente asociada con los aspectos íntimos de su interacción sexual que con el coito per se. El papel que juegan las parejas de las enfermas en cualquier etapa de la enfermedad es fundamental a la hora de promover una rehabilitación total y de consolidar la integridad de la relación (Die Trill, 2006).

A las alteraciones sexuales secundarias al tratamiento oncológico le suelen acompañar sentimientos de depresión, angustia, tristeza, y ansiedad que se unen a los temores y preocupaciones propios de una enfermedad que amenaza la vida. La pérdida del deseo sexual y la reducción en las sensaciones placenteras son propias de los trastornos depresivos. Los cuadros ansiosos interfieren igualmente en el funcionamiento sexual. De ahí la importancia de valorar ambos y de identificar su papel en la alteración sexual que experimenta el paciente (Tabano, Condosta y Coons, 2002).

\section{MATERNIDAD E INFERTILIDAD}

Entre las posibles secuelas del cáncer o de sus tratamientos se encuentra la infertilidad. En algunos pacientes, después de la cirugía o de la administración de quimioterapia o radioterapia contra el cáncer se produce un periodo de relativa esterilidad que puede durar desde varios meses a varios años.
En ocasiones se sugiere a la paciente que posponga la maternidad hasta pasado un tiempo tras la finalización del tratamiento, generalmente, con el fin de evitar embarazos en periodos de mayor riesgo de recidivas de la enfermedad. Incluso en otros casos la esterilidad que se produce puede ser irreversible. La esterilidad constituye un problema importante en supervivientes de linfoma de Hodgkin, entre otros, debido a los componentes de la quimioterapia administrada (por ejemplo, procarbazina) y a que un gran número de ellos reciben también irradiación en sus ovarios (Biasoli et al., 2012).

La infertilidad producida por el cáncer o sus tratamientos puede suponer un problema importante en enfermos jóvenes que han ido posponiendo la maternidad o la paternidad hasta tener relaciones de pareja o trabajos estables, o por cualquier otro motivo. Sentimientos de auto-reproche y de culpabilidad por haber dado prioridad a otros valores diferentes a la maternidad son frecuentes en estos casos. El malestar emocional que acompaña a las mujeres infértiles a causa del cáncer o de sus tratamientos suele estar caracterizado por angustia, ansiedad y una profunda tristeza por la pérdida de la capacidad para reproducirse, pérdida que se añade a otras producidas también por la situación médica. Los temores a que la incapacidad para reproducirse repercutan en la relación de pareja son igualmente frecuentes. Temor al abandono por parte de la pareja sana que ha podido haber deseado tener hijos, o temor a no encontrar una pareja estable por no poder reproducirse suelen estar presentes en muchas mujeres. Sentimientos de culpa suelen estar asociados a la responsabilidad que siente la mujer de no ser solo ella quien se ve obligada a privarse de la maternidad, sino también su pareja (Die Trill, 2004).

La capacidad para reproducirse suele estar asociada a múltiples sentimientos asociados a la maternidad y la femineidad, y en algunos casos, a la propia valía personal y auto-estima. Algunas mujeres que ven truncada su capacidad para engendrar pueden sentirse "menos mujeres", o incluso "menos personas" si no pueden satisfacer sus deseos de ser madres. La incapacidad para reproducirse está asociada a la inhibición del deseo sexual, a la evitación de las relaciones sexuales y a un interés reducido en la actividad sexual cuando la mujer se percibe a sí misma como menos atractiva sexualmente a consecuencia de la infertilidad. En los casos en los que se ha inducido una menopausia prematura como consecuencia del tratamiento contra el cáncer, a la incapacidad para reproducirse se añaden los síntomas físicos de la menopausia tales 
como irritabilidad, labilidad afectiva (esto es, cambios de humor repentinos), sofocos y sudores, insomnio, agitación nerviosa, etc.. Menopausia e infertilidad se unen aquí para producir un mayor número de reacciones emocionales negativas tales como ansiedad y depresión. En muchos casos las mujeres que han padecido determinados tipos de tumores no son candidatas adecuadas para recibir terapia hormonal sustitutoria con lo cual los síntomas desarrollados son difíciles de controlar pudiendo alterar significativamente su calidad de vida (Die Trill, 2004).

\section{LAS RELACIONES MATERNO-FILIALES Y EL IMPAC- TO DEL CÁNCER MATERNO SOBRE LOS HIJOS}

Se ha estudiado aunque no de forma especialmente extensa el impacto que tiene el cáncer materno sobre la relación con los hijos, siendo el foco central de estas investigaciones las mujeres con cáncer de mama. Las madres enfermas no siempre son conocedoras del proceso psicológico por el que están pasando sus hijos debido al cáncer de mama que ellas sufren (Zhalis y Lewis, 1998). Utilizan las preguntas que sus hijos les hacen sobre la enfermedad como estímulo para iniciar conversaciones con ellos sobre el cáncer, evitando diálogo con ellos cuando el niño se muestra retraído, en silencio o evita preguntar (Shands, Lewis y Zahlis, 2000). Durante las fases iniciales del cáncer las madres quieren ayudar a sus hijos y se preocupan enormemente por ellos, pero suelen experimentar demasiados síntomas físicos y estar excesivamente angustiadas con la enfermedad como para poder ejercer su rol de madre como lo ha hecho anteriormente. Con frecuencia reportan que quisieran proporcionar a sus hijos maneras eficaces de enfrentarse al cáncer pero que desconocen cómo hacerlo y cómo hablar con ellos sin aumentar su nivel de preocupación o distress. Con frecuencia se resisten a hablar abierta y claramente sobre la enfermedad con sus pequeños, desde su instinto de protección hacia ellos. Debido a su estado de vulnerabilidad física, en muchas ocasiones reportan no sentirse capaces de gestionar el estado afectivo de los niños (Zhalis y Lewis, 1998).

Las madres solteras pueden tener un riesgo añadido a la hora de ayudar a sus hijos a enfrentarse a su enfermedad. En algunos estudios se ha demostrado que estas madres enfermas dependen de sus hijos como una de sus principales fuentes de apoyo, compartiendo con ellos confidencias sobre su enfermedad y dependiendo de ellos cuando requerían asistencia inmediata (Lewis et al., 1996), sobrecargándoles emocionalmente con frecuencia.
Los hijos de las enfermas suelen hacer lo mismo que sus padres hacen con ellos, protegen a la enferma de la angustia que consideran pueden generar en sus madres al hablar o preguntar sobre el cáncer. Por ello tienden a reprimir o esconder sus pensamientos, temores y sentimientos en un intento de proteger a la paciente y no causar más tensión en la relación con ella (Issel, Ersek y Lewis, 1990).

Las madres con cáncer que tienen el estado de ánimo deprimido experimentan más dificultades para ejercer su rol de madre y para impartir disciplina adecuadamente sobre sus hijos. El estado de ánimo deprimido produce una visión negativa acentuada sobre la enfermedad y causa que la familia funcione menos eficazmente como unidad (Lewis y Hammond, 1992). Incluso se ha observado que la auto-estima de los hijos de pacientes con cáncer de mama puede sufrir debido a que internalizan los cambios en la auto-valía que sus madres experimentan como consecuencia de su dependencia, de la alteración en el funcionamiento y en el aspecto físico que experimentan como consecuencia del cáncer, y en la dificultad que muestran para mantener sus roles como lo hacían previamente a la enfermedad (Armsden y Lewis, 1994).

No es de sorprender que la investigación demuestre que el distress emocional es mayor en hijos del mismo sexo que el progenitor enfermo. Las hijas adolescentes son quienes más distress experimentan cuando su madre está enferma, comparadas con otros grupos de niños y jóvenes adultos con un progenitor enfermo (Compas et al., 1994). Wellisch y sus colegas observaron que mujeres que habían vivido el cáncer de mama materno durante su adolescencia (Wellisch et al., 1992), estaban peor adaptadas emocionalmente que aquéllas que eran de menor edad cuando su madre enfermó. Dos posibles explicaciones se pueden aportar: una es la creciente identificación que se produce durante la adolescencia, con el progenitor del mismo sexo, y que se puede ver alterada durante la enfermedad de la madre en las adolescentes. Otra posible explicación es la mayor demanda que recae sobre la adolescente para cubrir el rol de la madre cuando esta está enferma.

\section{MUJERES Y RIESGO GENÉTICO}

Existe muy poca literatura, y desde luego ningún estudio en profundidad, sobre el impacto que tiene sobre la mujer, transmitir una mutación genética que predisponga a desarrollar cáncer. Sin embargo, hay mucha información clínica anecdótica sobre los profundos sentimientos de culpa que siente la mujer una 
vez que ha aprendido que su hijo/a es portador/a de una mutación genética que ella misma tiene. Al fin y al cabo, los padres sienten un gran orgullo cuando observan las características positivas heredadas de sus hijos: "Tiene mis mismos ojos azules...". Algunas preguntas surgen en este contexto: ¿cuáles serán las consecuencias emocionales sobre la madre cuando conciba a un hijo sabiendo que es portadora de una mutación genética que le pone en riesgo a este? ¿Se sentirá incluso más culpable si el hijo desarrolla cáncer posteriormente? La decisión de no tener hijos es una decisión extrema, especialmente en los casos en los que la predisposición heredada existe en la edad adulta y cuando existe alguna promesa de que la prevención o el tratamiento pueden mejorar para cuando el hijo/a pueda haber desarrollado la enfermedad.

Mujeres que no tienen pareja en el momento en el que se les plantea el screening genético se enfrentan al temor de encontrar una pareja en el futuro que acepte el riesgo elevado de cáncer en su familia o en ella misma en el caso de que resulte ser portadora de una mutación (Patenaude, 2005). Mujeres homosexuales con historias significativas de cáncer de mama y ovario en sus familias tienen elevados niveles de preocupación y un gran interés en las pruebas genéticas (Durfy et al., 1999). La comunicación con su familia puede verse distorsionada por la falta de aceptación por parte de esta, de la orientación sexual de la mujer. Además, algunos miembros de la familia pueden confundir la heredabilidad del riesgo genético con la de la condición sexual, lo cual puede interferir aún más en el proceso de comunicación tan necesario. A esto se añade la necesidad de modificar ocasionalmente, la decisión acerca de quién quedará embarazada en la relación de pareja, en el caso de que una de ellas sea portadora de una mutación, pudiendo tener esto implicaciones importantes en los aspectos emocionales de la relación (Patenaude, 2005).

\section{CONCLUSIONES}

Es evidente lo importante que es tener en cuenta las diferencias de género cuando se estudia el impacto del cáncer sobre las personas. Las mujeres sufren alteraciones significativas en la imagen corporal y femineidad, así como en su sexualidad como consecuencia del cáncer. Cuestiones relacionadas con la maternidad, la auto-valía y la incapacidad de reproducirse tendrán un impacto significativo sobre su calidad de vida y su estado emocional. El cáncer afectará no solo a su adaptación psicológica global, sino a las relaciones de pareja y materno-filiales. Muchas se tienen que enfrentar además, a la posibilidad de ser transmisoras de una predisposición genética que puede influir en su decisión para tener hijos. A pesar de todo ello, la mujer generalmente cuenta con unos recursos personales significativos y potentes, en ocasiones específicos de la mujer, para afrontar estas alteraciones de formas que le permiten recuperar el máximo de su funcionamiento previo en muchos casos. No obstante, son necesarios más estudios centrados específicamente en la mujer, que identifiquen con más detalle sus necesidades psicosociales específicas con el fin de poder desarrollar programas concretos que tengan en cuenta las diferencias de género en el afrontamiento de la enfermedad oncológica, en el proceso de toma de decisiones médicas y en las variables necesarias para la optimización de su calidad de vida.

\section{BIBLIOGRAFÍA}

Algars, M., Santtila, P., Varjonen, M., Witting, K., Johansson, A., Jern, P. et al. (2009). The adult body: how age, gender, and body mass index are related to body image. Journal of Aging and Health, 21, 8, pp. 1112-1132. DOI: 21/8/1112 [pii]10.1177/0898264309348023.

Amsterdam, A. y Krychman, M. L. (2006). Sexual dysfunction in patients with gynecologic neoplasms: a retrospective pilot study. Journal of Sexual Medicine, 3, 4, pp. 646-649. DOI: JSM204 [pii]10.1111/ j.1743-6109.2006.00204.x.

Armsden, G. C. y Lewis, F. M. (1994). Behavioral adjustment and self-esteem of schoolage children of women with breast cancer. Oncology Nursing Forum, 21, 1, pp. 39-45.
Ashing-Giwa, K. T., Padilla, G., Tejero, J., Kraemer, J., Wright, K., Coscarelli, A. et al. (2004). Understanding the breast cancer experience of women: a qualitative study of African American, Asian American, Latina and Caucasian cancer survivors. Psychooncology, 13, 6, pp. 408-428. DOI: 10.1002/pon.750.

Biasoli, I., Falorio, S., Luminari, S., Spector, N. y Federico, M. (2012). Fertility in female survivors of Hodgkin's lymphoma. Revista Brasileira de Hematologia e Hemoterapia, 34, 1, pp. 48-53. DOI: 10.5581/1516-8484.20120014.

Carmack Taylor, C. L., Basen-Engquist, K., Shinn, E. H. y Bodurka, D. C. (2004). Predictors of Sexual Functioning in Ovar- ian Cancer Patients. Journal of Clinical Oncology, 22, 5, pp. 881-889. DOI: 10.1200/jco.2004.08.150.

Cash, T. F., Melnyk, S. E. y Hrabosky, J. I. (2004). The assessment of body image investment: an extensive revision of the appearance schemas inventory. International Journal of Eating Disorders, 35, 3, pp. 305-316. DOI: 10.1002/ eat.10264.

Compas, B. E., Worsham, N. L., EppingJordan, J. E., Grant, K. E., Mireault, G., Howell, D. C. et al. (1994). When mom or dad has cancer: markers of psychological distress in cancer patients, spouses, and children. Health Psychology, 13, 6, pp. 507-515. 
Diaz Morfa, J. (2008). Alteraciones sexuales y conflictos de pareja en oncología. En Die Trill, M. (ed.). Monografías de Oncología Médica . Madrid: You \& Us, pp. 87-110.

Die Trill, M. (2004). Infertilidad y cambios en la imagen corporal. Barcelona: Laboratorios Esteve.

Die Trill, M. (2006). Cáncer y sexualidad. La vida sigue. Barcelona: Laboratorios Esteve.

Durfy, S. J., Bowen, D. J., McTiernan, A. Sporleder, J. y Burke, W. (1999). Attitudes and interest in genetic testing for breast and ovarian cancer susceptibility in diverse groups of women in western Washington. Cancer Epidemiology, Biomarkers and Prevention, 8, 4 Pt 2, pp. 369-375.

Grassi, L., Malacarne, P., Maestri, A. y Ramelli, E. (1997). Depression, psychosocial variables and occurrence of life events among patients with cancer. Journal of Affective Disorders, 44, 1, pp. 21-30. DOI: S0165-0327(97)014456 [pii].

Hann, D., Baker, F., Denniston, M., Gesme, D., Reding, D., Flynn, T. et al. (2002). The influence of social support on depressive symptoms in cancer patients: age and gender differences. Journal of Psychosomatic Research, 52, 5, pp. 279283. DOI: S0022399901002355 [pii].

Issel, L. M., Ersek, M. y Lewis, F. M. (1990). How children cope with mother's breast cancer. Oncology Nursing Forum, 17, 3 Suppl, pp. 5-12; discussion 12-13.

Kiss, A. y Meryn, S. (2001). Effect of sex and gender on psychosocial aspects of prostate and breast cancer. British Medical Journal, 323, 7320, pp. 1055-1058.

Klemm, P. (2012). Effects of online support group format (moderated vs peer-led) on depressive symptoms and extent of participation in women with breast cancer. Computers, Informatics, Nursing, 30, 1, pp. 9-18. DOI: 10.1097/NCN.0b013e3182 343efa00024665-201201000-00004 [pii].
Klemm, P., Bunnell, D., Cullen, M., Soneji, R., Gibbons, P. y Holecek, A. (2003). Online cancer support groups: a review of the research literature. Computers, Informatics, Nursing, 21, 3, pp. 136-142.

Krok, J., Baker, T. y McMillan, S. (2013). Sexual activity and body image: examining gender variability and the influence of psychological distress in cancer patients. Journal of Gender Studies, 22, 4, pp. 409-422. DOI: 10.1080/09589236.2012.708828.

Lee, C. O. (1997). Quality of life and breast cancer survivors. Psychosocial and treatment issues. Cancer Practice, 5, 5, pp. 309-316.

Lewis, F. M. y Hammond, M. A. (1992). Psychosocial adjustment of the family to breast cancer: a longitudinal analysis. Journal of the American Medical Womens Association, 47, 5, pp. 194-200.

Lewis, F. M., Zahlis, E. H., Shands, M. E., Sinsheimer, J. A. y Hammond, M. A. (1996). The functioning of single women with breast cancer and their school-aged children. Cancer Practice, 4, 1, pp. 15-24.

Li, C. C. y Rew, L. (2010). A feminist perspective on sexuality and body image in females with colorectal cancer: an integrative review. Journal of Wound Ostomy and Continence Nursing, 37, 5, pp. 519-525. DOI: 10.1097/WON.0b013e31 81edac2c00152192-201009000-00013 [pii].

Liu, S. (2010). Patterns of symptoms in women after gynecologic surgery. Oncology Nursing Forum, 37, 2, pp. 133-140.

Patenaude, A. F. (2005). Genetic testing for cancer. Psychological approaches for helping patients and families. Washington: American Psychological Association.

Shands, M. E., Lewis, F. M. y Zahlis, E. H. (2000). Mother and child interactions about the mother's breast cancer: an interview study. Oncology Nursing Forum, 27, 1, pp. 77-85.
Sloan, J. (1992). Control preferences for treatment decision making in cancer patients. Berkeley: Miles Pharmaceuticals.

Tabano, M., Condosta, D. y Coons, M. (2002). Symptoms affecting quality of life in women with gynecologic cancer. Seminars in Oncology Nursing, 18, 3, pp. 223-230.

Tekkis, P. P., Cornish, J. A., Remzi, F. H., Tilney, H. S., Strong, S. A., Church, J. M. et al. (2009). Measuring sexual and urinary outcomes in women after rectal cancer excision. Diseases of the Colon and Rectum, 52, 1, pp. 46-54. DOI: $\quad$ 10.1007/DCR.0b013e31819755 1e00003453-200901000-00008 [pii]

Tom, A. (1993). Women's lives complete: methodological concerns. Women, work and coping: a multidisciplinary approach to workplace stress. Montreal: McGill-Queen's University Press.

Volkers, N. (1999). In coping with cancer, gender matters. Journal of the National Cancer Institute, 91, 20, pp. 1712-1714.

Wellisch, D. K., Gritz, E. R., Schain, W., Wang, H. J. y Siau, J. (1992). Psychological functioning of daughters of breast cancer patients. Part II: Characterizing the distressed daughter of the breast cancer patient. Psychosomatics, 33, 2, pp. 171-179. DOI: S0033-3182(92)71993-8 [pii]10.1016/S0033-3182(92)71993-8.

Wizemann, T. M. y Pardue, M. L. (eds.) (2001). Exploring the biological contributions to human health. Does sex matter? Washington: National Academies Press.

Woertman, L. y van den Brink, F. (2012). Body image and female sexual functioning and behavior: a review. Journal of Sex Research, 49, 2-3, pp. 184-211. DOI: 10.1080/00224499.2012.658586.

Zhalis, E. H. y Lewis, F. M. (1998). The mother's story of the school-aged child experience with the mother's breast cancer. Journal of Psychosocial Oncology, 16, 2, pp. 25-43. 